\title{
A THEOREM ON ARBITRARY $J$-FRACTIONS
}

H. S. WALL

1. Introduction. We consider a $J$-fraction

$$
1 a_{1}^{2} \quad\left(a_{p} \neq 0\right),
$$

in which the coefficients $a_{p}$ and $b_{p}$ are any complex numbers, the $a_{p}$ being different from zero, and $z$ is a complex parameter. The system of linear equations

$$
\begin{aligned}
-a_{p-1} x_{p-1}+\left(b_{p}+z\right) x_{p}-a_{p} x_{p+1} & =0, \\
& p=1,2,3, \cdots ; a_{0}=1,
\end{aligned}
$$

can be solved for $x_{2}, x_{3}, x_{4}, \ldots$ uniquely in terms of arbitrarily chosen initial values $x_{0}$ and $x_{1}$. We denote by $X_{p}(z)$ and $Y_{p}(z)$ the solutions corresponding to $x_{0}=-1, x_{1}=0$ and $x_{0}=0, x_{1}=1$, respectively: $X_{0}(z)=-1, X_{1}(z)=0, Y_{0}(z)=0, Y_{1}(z)=1$. Then $X_{p+1}(z) / Y_{p+1}(z)$ is the $p$ th approximant of the $J$-fraction, and we have the determinant formula

$$
X_{p+1}(z) Y_{p}(z)-X_{p}(z) Y_{p+1}(z)=1 / a_{p}, \quad p=0,1,2, \cdots .
$$

The following theorem holds.

TheOREM OF INVARIABILITy. If the series

$$
\sum_{p=1}^{\infty}\left|X_{p}(z)\right|^{2}, \quad \sum_{p=1}^{\infty}\left|Y_{p}(z)\right|^{2}
$$

converge for a single value of the parameter $z$, then these series converge uniformly over every bounded domain of $z$.

This theorem was proved by Hellinger and Wall [3]. ${ }^{1}$ The uniformity of the convergence was not explicitly mentioned, but is contained

Presented to the Society, September 17, 1945; received by the editors December $26,1945$.

1 Numbers in brackets refer to the Bibliography at the end of the paper. 
in the fact that the sums $\sum_{p=1}^{m}\left|\zeta_{p}\right|^{2}$ of $[3$, p. 121] are uniformly bounded over every finite domain of $z^{*}$.

In the present note we have derived some consequences of the above theorem. It will be convenient to make the following definition.

Definition. The determinate case or the indeterminate case is said to hold for the $J$-fraction (1.1) according as at least one of the series

$$
\sum_{p=1}^{\infty}\left|X_{p}(0)\right|^{2}, \quad \sum_{p=1}^{\infty}\left|Y_{p}(0)\right|^{2}
$$

diverges, or both of these series converge, respectively.

We shall prove that if the indeterminate case holds for the $J$-fraction, and if the $J$-fraction converges for a single value of $z$, then it represents a meromorphic function of $z$ and converges except at the poles of this function. Hamburger [2] proved this theorem for $J$-fractions with real coefficients, and closely related theorems were proved by Hellinger and Wall [3] for $J$-fractions in which the $a_{p}$ are real and $I\left(b_{p}\right) \geqq 0$, and by Dennis and Wall [1] for positive definite $J$-fractions. If, in particular, $b_{p}=0, p=1,2,3, \cdots$, then, if we drop the factor $z$, the $J$-fraction can be thrown into the form

$$
\frac{1}{1} \quad\left(w=z^{2}\right) .
$$

We show that if the series

$$
\sum k_{2 p+1}, \quad \sum k_{2 p+1}\left(k_{2}+k_{4}+\cdots+k_{2 p}\right)^{2}
$$

are absolutely convergent and

$$
\lim _{p=\infty}\left|k_{2}+k_{4}+\cdots+k_{2 p}\right|=\infty,
$$

then the continued fraction (1.6) converges to a meromorphic function of $w$ or else diverges to $\infty$ for every $w$. If the series (1.7) are absolutely convergent and (1.8) fails to hold, then the continued fraction diverges by oscillation for every $w$. This theorem was proved by Hamburger [2] for the case where the $k_{p}$ are real, $k_{2 p} \neq 0$, and $k_{2 p+1}>0$.

2. Four entire functions. We define four polynomials $U_{n}(z), V_{n}(z)$ 
$P_{n}(z), Q_{n}(z)$ by means of the following formulas:

$$
\begin{aligned}
U_{n}(z) & =a_{n}\left[Y_{n}(0) X_{n+1}(z)-Y_{n+1}(0) X_{n}(z)\right] \\
V_{n}(z) & =a_{n}\left[Y_{n}(0) Y_{n+1}(z)-Y_{n+1}(0) Y_{n}(z)\right], \quad n=1,2,3, \cdots . \\
P_{n}(z) & =a_{n}\left[X_{n}(0) X_{n+1}(z)-X_{n+1}(0) X_{n}(z)\right], \\
Q_{n}(z) & =a_{n}\left[X_{n}(0) Y_{n+1}(z)-X_{n+1}(0) Y_{n}(z)\right]
\end{aligned}
$$

We find with the aid of the determinant formula (1.3) that these polynomials satisfy the identity

$$
P_{n}(z) V_{n}(z)-Q_{n}(z) U_{n}(z) \equiv 1 .
$$

We now put $x_{k}=X_{k}(z), p=n+1$, in (1.2), and get

$$
a_{n+1} X_{n+2}(z)=\left(b_{n+1}+z\right) X_{n+1}(z)-a_{n} X_{n}(z) \text {. }
$$

We multiply both members of this identity by $X_{n+1}(0)$, subtract $a_{n+1} X_{n+2}(0) X_{n+1}(z)$ from both members, and obtain

$$
\begin{aligned}
a_{n+1}\left[X_{n+1}(0) X_{n+2}(z)\right. & \left.-X_{n+2}(0) X_{n+1}(z)\right] \\
= & {\left[\left\{b_{n+1} X_{n+1}(0)-a_{n+1} X_{n+2}(0)\right\}+z X_{n+1}(0)\right] X_{n+1}(z) } \\
& \quad-a_{n} X_{n+1}(0) X_{n}(z) \\
= & a_{n}\left[X_{n}(0) X_{n+1}(z)-X_{n+1}(0) X_{n}(z)\right]+z X_{n+1}(0) X_{n+1}(z) .
\end{aligned}
$$

Hence, by (2.1), we have the first of the following relations:

$$
\begin{aligned}
& P_{n+1}(z)=P_{n}(z)+z X_{n+1}(0) X_{n+1}(z), \\
& Q_{n+1}(z)=Q_{n}(z)+z X_{n+1}(0) Y_{n+1}(z), \\
& U_{n+1}(z)=U_{n}(z)+z Y_{n+1}(0) X_{n+1}(z), \\
& V_{n+1}(z)=V_{n}(z)+z Y_{n+1}(0) Y_{n+1}(z) .
\end{aligned}
$$

The others may be obtained in a similar way. From these relations we now obtain immediately the following formulas.

$$
\begin{aligned}
& P_{n+1}(z)=+z \sum_{p=2}^{n+1} X_{p}(0) X_{p}(z), \\
& Q_{n+1}(z)=-1+z \sum_{p=2}^{n+1} X_{p}(0) Y_{p}(z), \\
& U_{n+1}(z)=1+z \sum_{p=2}^{n+1} Y_{p}(0) X_{p}(z), \\
& V_{n+1}(z)=z+z \sum_{p=2}^{n+1} Y_{p}(0) Y_{p}(z) .
\end{aligned}
$$


By the theorem of invariability $(\S 1)$ and formulas $(2.2),(2.3)$ we find at once by Schwarz's inequality that the following theorem is true.

THEOREM 2.1. Let the indeterminate case hold for the J-fraction (1.1). Then there exist four entire functions $u(z), v(z), p(z), q(z)$ such that

$$
p(z) v(z)-q(z) u(z)=1,
$$

and such that

$$
\begin{aligned}
& \lim _{n=\infty} U_{n}(z)=u(z), \quad \lim _{n=\infty} V_{n}(z)=v(z), \\
& \lim _{n=\infty} P_{n}(z)=p(z), \quad \lim _{n=\infty} Q_{n}(z)=q(z),
\end{aligned}
$$

uniformly over every bounded region of the z-plane.

3. Convergence theorem for $J$-fractions. By means of (2.1) and (1.3) we find that

$$
\begin{aligned}
& X_{n+1}(z)=X_{n+1}(0) U_{n}(z)-Y_{n+1}(0) P_{n}(z), \\
& Y_{n+1}(z)=X_{n+1}(0) V_{n}(z)-Y_{n+1}(0) Q_{n}(z) .
\end{aligned}
$$

Let $s_{n}=X_{n+1}(0) / Y_{n+1}(0)$. If $\lim _{n=\infty} s_{n}=s$, a finite number, and if the indeterminate case holds for the $J$-fraction, it then follows from Theorem 2.1 and the relations (3.1) that

$$
\lim _{n=\infty} \frac{X_{n+1}(z)}{Y_{n+1}(0)}=s u(z)-p(z), \quad \lim _{n=\infty} \frac{Y_{n+1}(z)}{Y_{n+1}(0)}=s v(z)-q(z),
$$

uniformly over every finite region. Since, by (2.4),

$$
[s v(z)-q(z)] u(z)-[s u(z)-p(z)] v(z)=1,
$$

it follows that the limits (3.2) cannot vanish for one and the same value of $z$. Therefore, for every value of $z$,

$$
\lim _{n=\infty} \frac{X_{n+1}(z)}{Y_{n+1}(z)}=\frac{s u(z)-p(z)}{s v(z)-q(z)},
$$

which is a meromorphic function of $z$, or else is $\infty$ for every $z$. If $\lim _{n=\infty} s_{n}=\infty$, then, for every value of $z$,

$$
\lim _{n=\infty} \frac{X_{n+1}(z)}{Y_{n+1}(z)}=\frac{u(z)}{v(z)}
$$

which again is a meromorphic function of $z$ or else is $\infty$ for every $z$. If the sequence $\left\{s_{n}\right\}$ has more than one limit-point, let $\lim _{n^{\prime}-\infty} s_{n^{\prime}}=s^{\prime}$, 
$\lim _{n^{\prime \prime}-\infty} s_{n^{\prime \prime}}=s^{\prime \prime}, s^{\prime} \neq s^{\prime \prime}$. Then, if $s^{\prime}$ and $s^{\prime \prime}$ are both finite,

$$
\begin{aligned}
& \lim _{n^{\prime}=\infty} \frac{X_{n^{\prime}+1}(z)}{Y_{n^{\prime}+1}(z)}=\frac{s^{\prime} u(z)-p(z)}{s^{\prime} v(z)-q(z)}, \\
& \lim _{n^{\prime \prime}=\infty} \frac{X_{n^{\prime \prime}+1}(z)}{Y_{n^{\prime \prime}+1}(z)}=\frac{s^{\prime \prime} u(z)-p(z)}{s^{\prime \prime} v(z)-q(z)} .
\end{aligned}
$$

These are unequal for every $z$ inasmuch as

$$
\left(s^{\prime} u-p\right)\left(s^{\prime \prime} v-q\right)-\left(s^{\prime \prime} u-p\right)\left(s^{\prime} v-q\right)=s^{\prime}-s^{\prime \prime} \neq 0,
$$

and therefore the $J$-fraction diverges by oscillation for every $z$. The same evidently holds if one of the limits $s^{\prime}, s^{\prime \prime}$ is $\infty$. From these considerations we conclude that the following theorem is true.

THEOREM 3.1. Let the indeterminate case hold for the J-fraction (1.1). If the $J$-fraction converges for a single value of $z$, then it represents $a$ meromorphic function of $z$ and converges except at the poles of that function. In terms of the entire functions of $\$ 2$,

$$
\lim _{n=\infty} \frac{X_{n+1}(z)}{Y_{n+1}(z)}=\frac{s u(z)-p(z)}{s v(z)-q(z)} \text { or } \frac{u(z)}{v(z)}
$$

according as

$$
\lim _{n=\infty} \frac{X_{n+1}(0)}{Y_{n+1}(0)}=s(\text { finite }) \text { or } \infty,
$$

respectively. If there is a single value of $z$ for which neither the J-fraction nor its reciprocal converges, then the J-fraction diverges by oscillation for every value of $z$.

4. Convergence theorem for $S$-fractions. A $J$-fraction (1.1) in which the coefficients $b_{p}$ are all equal to zero is called a Stieltjes fraction or $S$-fraction:

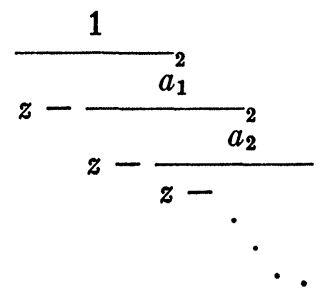

This has the property that its even part is a $J$-fraction in the variable $z^{2}$, multiplied by the factor $z$, namely, 


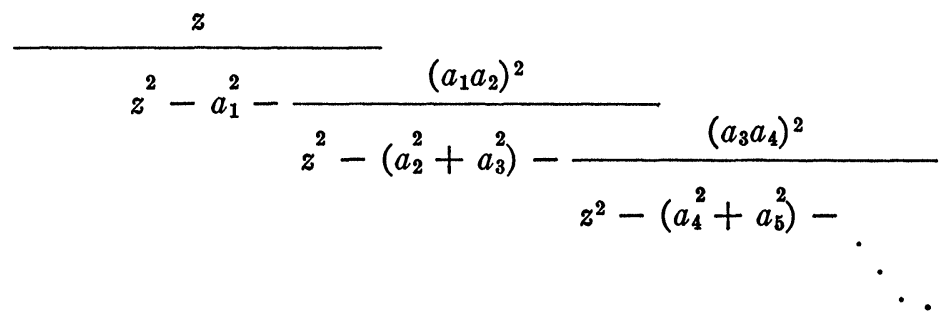

If we divide both (4.1) and (4.2) by $z$, and then make the change of variable $z^{2}=w$, these take the form

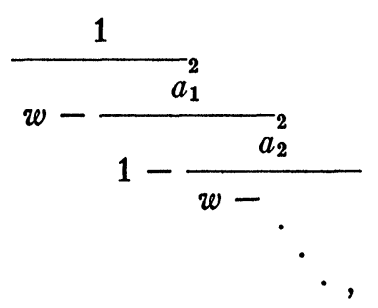

and

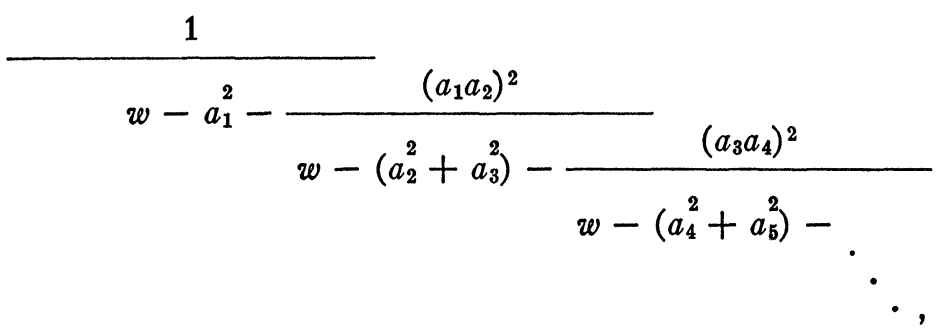

respectively. We shall apply Theorem 3.1 to the $J$-fraction (4.4) in order to obtain a convergence theorem for the $S$-fraction (4.3).

Let $G_{p}(w)$ and $H_{p}(w)$ denote the $p$ th numerator and denominator of (4.3), and let $A_{p}(w)$ and $B_{p}(w)$ denote the $p$ th numerator and denominator of (4.4). Then we have

$$
G_{2 p}(w)=A_{p}(w), \quad H_{2 p}(w)=B_{p}(w), \quad p=0,1,2, \cdots .
$$

Let

$$
\begin{array}{ll}
\sigma_{1}=a_{1} a_{2}, & \delta_{1}=-a_{1}^{2}, \\
\sigma_{2}=a_{3} a_{4}, & \delta_{2}=-a_{1}^{2}-a_{2}^{2}, \\
\sigma_{3}=a_{5} a_{6}, & \delta_{3}=-a_{4}^{2}-a_{5}^{2},
\end{array}
$$


so that (4.4) becomes

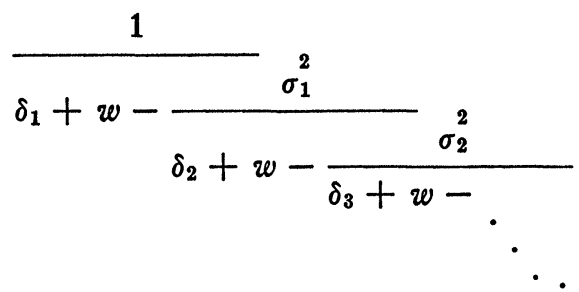

From (4.6) we readily find by mathematical induction that

$$
a_{2 p-1}^{2}=-\frac{B_{p}(0)}{B_{p-1}(0)}, \quad a_{2 p}^{2}=-\frac{\sigma_{p}^{2} B_{p-1}(0)}{B_{p}(0)}, p=1,2,3, \cdots,
$$

and

$$
B_{p}(0) \neq 0,
$$$$
p=1,2,3, \cdots \text {. }
$$

Conversely, if (4.7) is a $J$-fraction such that (4.9) holds, then it is the even part of an $S$-fraction (4.3) whose partial numerators are given by (4.8).

The odd numerators and denominators of the $S$-fraction (4.3) can be expressed in terms of the numerators and denominators of (4.7) by means of the formulas

$$
\begin{aligned}
& G_{2 p-1}(w)=\frac{B_{p-1}(0) A_{p}(w)-B_{p}(0) A_{p-1}(w)}{B_{p-1}(0)}, \\
& H_{2 p-1}(w)=\frac{B_{p-1}(0) B_{p}(w)-B_{p}(0) B_{p-1}(w)}{B_{p-1}(0)}, p=1,2,3, \cdots .
\end{aligned}
$$

These may be verified immediately by means of the recurrence formula $G_{2 p}(w)=G_{2 p-1}(w)-a_{2 p-1}{ }^{2} G_{2 p-2}(w)$, and the like relation for $H_{2 p}(w)$, if we use (4.5) and (4.8). It will be observed that the first two polynomials (2.1), formed for the $J$-fraction (4.7), differ from (4.10) only by a constant factor:

$$
\frac{G_{2 p-1}(w)}{H_{2 p-1}(w)}=\frac{U_{p}(w)}{V_{p}(w)}, \quad p=1,2,3, \cdots .
$$

If we make the substitution

$$
a_{p}^{2}=-\frac{1}{k_{p} k_{p+1}}, \quad p=1,2,3, \cdots ; k_{1}=1,
$$

in (4.3), the latter becomes 


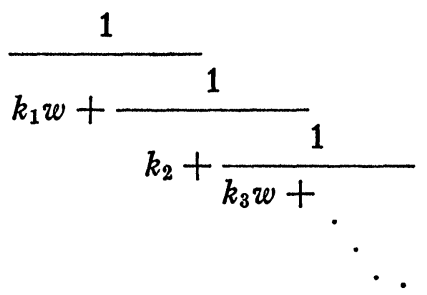

The $p$ th numerator and denominator of (4.13) are

$$
k_{1} k_{2} \cdots k_{p} G_{p}(w) \text { and } k_{1} k_{2} \cdots k_{p} H_{p}(w),
$$

respectively.

We shall now prove the following theorem.

THEOREM 4.1. Let $k_{1}, k_{2}, k_{3}, \ldots$ be complex numbers different from zero such that the series

$$
\sum k_{2 p+1}, \quad \sum k_{2 p+1}\left(k_{2}+k_{4}+\cdots+k_{2 p}\right)^{2}
$$

are absolutely convergent, and let

$$
\lim _{p=\infty}\left(x_{2}+k_{4}+\cdots+k_{2 p}\right)=\infty .
$$

Then, for each value of w, the S-fraction (4.13) converges, or else its reciprocal converges to the value 0 . If the series (4.14) converge absolutely and (4.15) fails to hold, then neither the S-fraction nor its reciprocal converges for a single value of $w$. If the $S$-fraction converges for one value of w, then its value is a meromorphic function of w to which it converges uniformly over every bounded closed region containing none of the poles of the function.

Proof. The polynomials $X_{p+1}(w)$ and $Y_{p+1}(w)$ for the $J$-fraction (4.7) are given by

$$
X_{p+1}(w)=\frac{A_{p}(w)}{\sigma_{1} \sigma_{2} \cdots \sigma_{p}}, \quad Y_{p+1}(w)=\frac{B_{p}(w)}{\sigma_{1} \sigma_{2} \cdots \sigma_{p}} .
$$

By (4.5), (4.6) and (4.12) we then obtain

$$
\begin{aligned}
& \left|X_{p+1}(0)\right|^{2}=\left|G_{2 p}(0) \cdot k_{1} k_{2} \cdots k_{2 p}\right|^{2} \cdot\left|k_{2 p+1}\right|, \\
& \left|Y_{p+1}(0)\right|^{2}=\left|H_{2 p}(0) \cdot k_{1} k_{2} \cdots k_{2 p}\right| \cdot\left|k_{2 p+1}\right| .
\end{aligned}
$$

Since $k_{1} k_{2} \cdots k_{p} G_{p}(w)$ and $k_{1} k_{2} \cdots k_{p} H_{p}(w)$ are the $p$ th numerator and denominator, respectively, of (4.13), we readily find by mathematical induction that

$G_{2 p}(0) \cdot k_{1} k_{2} \cdots k_{2 p}=k_{2}+k_{4}+\cdots+k_{2 p}, H_{2 p}(0) \cdot k_{1} k_{2} \cdots k_{2 p}=1$, 
and consequently

$$
\begin{aligned}
\left|X_{p+1}(0)\right|^{2} & =\left|k_{2 p+1}\left(k_{2}+k_{4}+\cdots+k_{2 p}\right)^{2}\right|, \\
\left|Y_{p+1}(0)\right|^{2} & =\left|k_{2 p+1}\right| .
\end{aligned}
$$

From Theorem 3.1 we therefore conclude that when the series (4.14) converge absolutely, then, by (4.11):

$$
\lim _{p=\infty} \frac{G_{2 p-1}(w)}{H_{2 p-1}(w)}=\frac{u(w)}{v(w)},
$$

where $u(w)$ and $v(w)$ are entire functions, provided $v(w) \neq 0$. Inasmuch as $u(w) \neq 0$ when $v(w)=0$, we conclude that for any $w$, the sequence of odd approximants of the $S$-fraction, or else the sequence of reciprocals of these approximants, must converge. By Theorem 3.1 and (4.5),

$$
\lim _{p=\infty} \frac{G_{2 p}(w)}{H_{2 p}(w)}=\frac{u(w)}{v(w)}
$$

if and only if $\lim _{p=\infty}\left|A_{p}(0) / B_{p}(0)\right|=\lim _{p=\infty}\left|X_{p+1}(0) / Y_{p+1}(0)\right|=\infty$. But, by (4.16),

$$
\left|\frac{X_{p+1}(0)}{Y_{p+1}(0)}\right|=\left|k_{2}+k_{4}+\cdots+k_{2 p}\right| .
$$

Therefore, if (4.15) holds, then the $S$-fraction or its reciprocal converges. If (4.15) fails to hold, then the $S$-fraction and its reciprocal diverge for every $w$. If the $S$-fraction converges for a single value of $w$, then its value is the meromorphic function $u(w) / v(w)$. The convergence is clearly uniform over every closed bounded region containing none of the poles of this function.

\section{BIBLIOGRAPHY}

1. J. J. Dennis and H. S. Wall, The limit-circle case for a positive definite J-fraction, Duke Math. J. vol. 12 (1945) pp. 255-273.

2. H. Hamburger, Über eine Erweiterung des Stieltjesschen Momentenproblems, Math. Ann. vol. 81 (1920) pp. 235-319; vol. 82 (1921) pp. 120-164 and 168-187.

3. E. D. Hellinger and H. S. Wall, Contributions to the analytic theory of continued fractions and infinite matrices, Ann. of Math. (2) vol. 44 (1943) pp. 103-127.

ILLINOIS INSTITUTE OF TEChNOLOGY 